\title{
Stacking-fault energies in simple metals: applications to BCC metals
}

\author{
John F Devlin \\ University of Michigan-Dearborn. 4901 Evergreen Road. Dearborn. Michigan 48128. \\ USA
}

Received 28 April 1981, in final form 19 June 1981

\begin{abstract}
We present a general method for calculating the stacking-fault energy in simple metals, and then we apply this to the $\{112\}$ faults in body-centred cubic (BCC) metals. Our method contains no approximations for a given wavenumber characteristic (or equivalently the pair potential). Our results show that metastable faults do indeed exist in the simple BCC metals ( $\mathrm{Li}, \mathrm{Na}$. K, Rb. Cs. Ca, Sr, Ba), but the currently available potentials do not yield sufficiently accurate stacking-fault energies because they do not predict BCC as the lowest energy phase.
\end{abstract}

\section{Introduction}

The stacking-fault energy problem has been extensively studied in the past. Most of these efforts have been directed towards the face-centred cubic (FCC) metals (see Simon 1979 for a review). The methods used have either been the direct space method or the reciprocal space method. In the direct space method the pair potential is summed over all pairs of ions that cross the stacking-fault plane. Because the known theoretical pair potentials (which are generated from pseudopotential theory) have a long-range oscillatory tail, convergence problems usually arise and hence cloud the reliability of the results. This convergence problem does not arise in the reciprocal space method because the Fourier transform of the pair potential, the wavenumber characteristic, is short-range. The only approximation made in the reciprocal space method is in the particular wavenumber characteristic used. Hence, this method is preferred for these long-range potentials. In the case of short-range potentials, which are empirically generated, the direct space method is preferable (see Vitek 1968 for examples of this method).

In an earlier paper (Devlin 1974), we presented the reciprocal space method for the stacking-fault energy of the simple FCC and hexagonal close-packed (HCP) metals. The particular method used there depended heavily on the special symmetry of the FCC and HCP stacking faults. In this paper, we present a more generalised expression for calculating the stacking-fault energy within the reciprocal space method. Our method is valid for any stacking fault in which the half-crystals on each side of the fault are translationally invariant. As such, this assumption of translational invariance of each half-crystal excludes us from considering relaxation effects in the vicinity of the boundary. In the case of FCC and HCP faults, our more general expression reduces to the usual result. 
We have applied our new method to investigating the existence of $\{112\}$ faults in body-centred cubic (BCC) metals. This is a rather stringent test of the method and of the potentials used because, unlike the FCC and HCP faults, the nearest-neighbour distances change across the BCC fault plane. Some controversy remains as to whether such faults even exist. The theoretical calculations have been split on this issue: Vitek (1968), Basinski et al (1970) and Rao (1975) do not find stable stacking faults on the \{112\} planes whereas Eichler and Pegel (1969) do. All four calculations used the direct space method and so are possibly suspect to convergence problems, depending on whether their potential is short- or long-range. When our reciprocal space method is applied to these $\{112\}$ faults using long-range potentials, we do find metastable fault configurations in the vicinity of the hypothesised faults. However, for the metals concerned in this work the numerical results yielded stacking-fault energies which were negative for certain configurations, indicating that the BCC phase itself is unstable. Hence, none of the potentials currently available are reliable enough to show the BCC phase as being preferable over all other phases.

Specifically, we have calculated the twin and intrinsic stacking-fault energies of the metals $\mathrm{Li}, \mathrm{Na}, \mathrm{K}, \mathrm{Rb}, \mathrm{Cs}, \mathrm{Ca}, \mathrm{Sr}$ and $\mathrm{Ba}$ using the model pseudopotential of Appapillai and Williams (1973, to be referred to as $\mathrm{AW}$ ) and in the case of $\mathrm{Ca}, \mathrm{Sr}$ and $\mathrm{Ba}$, we also used the $d$ state pseudopotential of Moriarty (1972). Both of these potentials include the many-electron correlation effects known to be important in structural studies. The $\mathrm{Aw}$ potential for $\mathrm{Be}, \mathrm{Mg}$ and $\mathrm{Al}$ yielded good agreement between theory and experiment for the FCC and HCP faults. Hence, we feel that the AW potentials are the best pseudopotentials currently available for structural studies of the simple metals.

\section{General method}

In pseudopotential theory the structure-dependent part of the cohesive energy (per crystal ion) can be written as

$$
E=\sum_{q}^{\prime}|S(q)|^{2} F(q)
$$

where the prime indicates summing over all wavevectors, $q$, except $q=0$. The function $F(q)$ is the Fourier transform of the pair potential between the ions. $S(q)$ is the static structure factor of the lattice

$$
S(q)=\frac{1}{N} \sum_{\boldsymbol{R}_{\mathrm{i}}} \exp \left(-\mathrm{i} \boldsymbol{q} \cdot \boldsymbol{R}_{\mathrm{i}}\right)
$$

where the $\boldsymbol{R}_{\mathrm{i}}$ are the $N$ ionic positions. To obtain the stacking-fault energy of just a single stacking fault, we have to take the difference in energy between two crystals: one faulted and one unfaulted. Then the stacking-fault energy (per crystal ion) is given by

$$
E_{\mathrm{sf}}=\sum_{q}^{\prime} F(q)\left(\left|S_{\mathrm{faulted}}(q)\right|^{2}-\left|S_{\text {unfaulted }}(q)\right|^{2}\right) .
$$

In order to perform this delicate subtraction to obtain the stacking-fault energy per fault ion, we describe the crystal structure in the following manner. Each ion is 
associated with a particular unit cell. The unit cells are chosen so that the lattice of unit cells is translationally invariant across the fault plane. The only difference in the unit cells across the fault plane is in their internal structure. This lattice of unit cells is frequently called the coincidence-site lattice (see Bollmann 1970 for a fuller discussion of these lattices). Then an ionic position is described with two labels:

$$
\boldsymbol{R}_{\mu v}=\boldsymbol{R}_{\mu}+\boldsymbol{r}_{v}
$$

where the vector $\boldsymbol{R}_{\mu}$ describes the particular unit-cell origin and the $\boldsymbol{r}_{\mathrm{v}}$ locates the individual ion within the unit cell. The set of $\left\{\boldsymbol{R}_{u}\right\}$ forms the coincidence lattice. The set of $r$ would be different on each side of the fault plane. Then

$$
S(\boldsymbol{q})=\frac{1}{N}\left(\sum_{\mu} \exp \left(-\mathrm{i} \boldsymbol{q} \cdot \boldsymbol{R}_{u}\right)\right)\left(\sum_{v} \exp \left(-\mathrm{i} \boldsymbol{q} \cdot \boldsymbol{r}_{v}\right)\right) .
$$

Now, referring to figure 1 , we consider our fault plane to be perpendicular to the $z$ axis. Then every vector $\boldsymbol{R}_{\mu}$ can be written as $\boldsymbol{R}_{\mu}=\boldsymbol{R}_{\mu \perp}+R_{\mu z} \hat{z}$, where the $\boldsymbol{R}_{\mu \perp}$ is in the $x-y$ plane. Similarly, we write $q=q_{\perp}+q_{z} \hat{z}$. Then the sum over all unit cells in a single $x-y$ plane is easily performed:

$$
\sum_{\substack{\mu \\ \text { (in one plane) }}} \exp \left(-\mathrm{i} \boldsymbol{q}_{\perp} \cdot \boldsymbol{R}_{\mu_{\perp}}\right)=N_{p} \delta_{q_{+}} \cdot G_{-}
$$

where the $G_{\perp}$ are the reciprocal lattice vectors of the $x-y$ plane, $N_{\mathrm{p}}$ is the number of ions in one plane, and $\delta$ is the usual Krönecker delta function. Now we define partial-structure factors for the half-crystals

$$
\begin{aligned}
& S_{1}\left(q_{z}\right)=\frac{1}{N_{z}} \sum_{\substack{u \\
(\text { left })}} \exp \left(-\mathrm{i} q_{z} R_{u z}\right) \\
& S_{2}\left(q_{z}\right)=\frac{1}{N_{z}} \sum_{\substack{u \\
\text { (right) }}} \exp \left(-\mathrm{i} q_{z} R_{u z}\right)
\end{aligned}
$$

where the sum for $S_{1}\left(S_{2}\right)$ is performed over all unit-cell origins along the $z$ axis that are to the left (right) of the fault and $N_{z}$ is the total number of unit-cell planes parallel to the fault plane. Finally, we define unit-cell structure factors

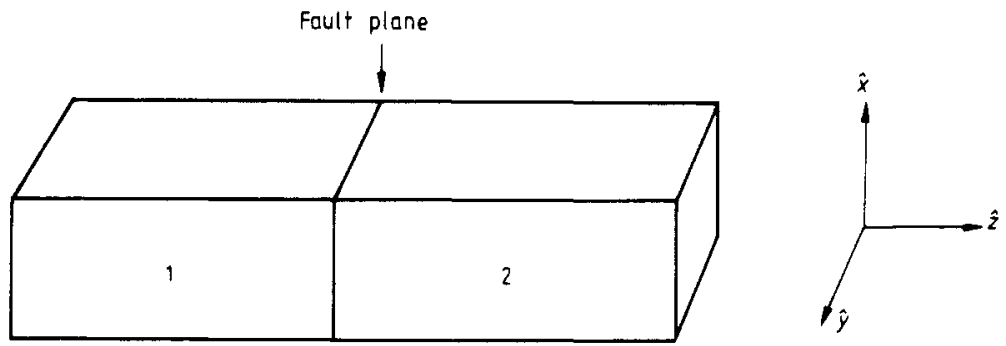

Figure 1. An idealisation of a faulted crystal. The crystal structure is assumed to be perfect on each side of the fault and the crystal is assumed perfectly long in the z direction which is normal to the fault plane. 


$$
\begin{aligned}
& f_{1}(\boldsymbol{q})=\frac{1}{N_{0}} \sum_{\substack{1 \\
(1 \mathrm{k}(t)}} \exp \left(-\mathrm{i} \boldsymbol{q} \cdot \boldsymbol{r}_{\mathrm{v}}\right) \\
& f_{2}(\boldsymbol{q})=\frac{1}{N_{\mathrm{c}}} \sum_{\substack{\boldsymbol{v} \\
(\text { right) }}} \exp \left(-\mathrm{i} \boldsymbol{q} \cdot \boldsymbol{r}_{\mathrm{v}}\right)
\end{aligned}
$$

where, again. the indices 1 and 2 refer to summations on the respective sides of the fault. Since $N=N_{\mathrm{p}} N_{z} N_{\mathrm{c}}$ the total structure factor for a faulted crystal is of the form

$$
S_{\text {fivulted }}(q)=\delta_{q_{-}, G}\left(S_{1}\left(q_{z}\right) f_{1}(q)+S_{2}\left(q_{z}\right) f_{2}(q)\right) .
$$

The structure factor for a perfect (unfaulted) crystal would then be

$$
S_{\text {unfiulted }}(q)=\delta_{q_{.} . G_{.}}\left(S_{1}\left(q_{z}\right)+S_{2}\left(q_{z}\right)\right) f_{1}(q)
$$

or

$$
S_{\text {unfuulted }}(q)=\delta_{q_{.} . G .}\left(S_{1}\left(q_{2}\right)+S_{2}\left(q_{z}\right)\right) f_{2}(q)
$$

since the unfaulted crystal may be of either $f_{1}$ or $f_{2}$ structure, both of which, of course, have the same energy. For symmetry reasons we will find it convenient to use a combination of equations $(7 a)$ and $(7 b)$ for the squared magnitude of $S_{\text {unfiulted }}$ :

$$
\left|\mathrm{S}_{\text {unfiullided }}(\boldsymbol{q})\right|^{2}=\frac{1}{2} \delta_{\boldsymbol{q}, \boldsymbol{G}}\left|S_{1}\left(q_{z}\right)+S_{2}\left(q_{z}\right)\right|^{2}\left(\left.\left|f_{1}(\boldsymbol{q})^{2}+\right| f_{2}(\boldsymbol{q})\right|^{2}\right) .
$$

Then the stacking-fault energy expression of equation (3) becomes

$$
E_{\checkmark i}=\sum_{q}^{\prime} \delta_{u_{1} . G} F(q)\left[S_{1} S_{2}^{*}\left(f_{1} f_{2}^{*}-\frac{1}{2}\left|f_{1}\right|^{2}-\frac{1}{2}\left|f_{2}\right|^{2}\right)+S_{1}^{*} S_{2}\left(f_{1}^{*} f_{2}-\frac{1}{2}\left|f_{1}\right|^{2}-\left.\frac{1}{2} f_{2}\right|^{2}\right)\right]
$$

where terms of the form $\left|S_{i}\right|^{2}\left|f_{j}\right|^{2}$ all drop out because each of the $f_{i}$ correspond to the same structure and hence have the same crystal energy.

We assume the $R_{u z}$ planes are spaced a distance $d$ apart and, if we label them with integers $n$, we have

$$
\begin{aligned}
& S_{1}\left(q_{z}\right)=\frac{1}{N_{z}} \sum_{n=\left(-N_{z} / 2\right)+1}^{0} \exp \left(-\mathrm{i} q_{z} n d\right) \\
& S_{2}\left(q_{z}\right)=\frac{1}{N_{z}} \sum_{n=1}^{N_{z} / 2} \exp \left(-\mathrm{i} q_{z} n d\right) .
\end{aligned}
$$

In the spirit of Hodges (1967) we introduce convergence factors into each of the sums:

$$
\begin{aligned}
& S_{1}\left(q_{z}\right)=\lim _{i \rightarrow 0^{-}} \frac{1}{N_{z}} \sum_{n} \sum_{\left(-N_{z} / 2\right)+1}^{0} \exp \left[-\mathrm{i}\left(q_{z}+\mathrm{i} i\right) n d\right] \\
& S_{2}\left(q_{z}\right)=\lim _{i \rightarrow 0^{+}} \frac{1}{N_{z}} \sum_{n=1}^{N_{z} / 2} \exp \left[-\mathrm{i}\left(q_{z}-\mathrm{i} z\right) n d\right] .
\end{aligned}
$$


The small parameter $\varepsilon$ is chosen so that for very large $N_{z}, i d N_{z} \gg 1$. Then the sums are easily performed to yield

$$
S_{1} S_{2}^{*}=\left(\frac{-1}{4 N_{z}^{2}}\right) \lim _{\rightarrow 0} \frac{1}{\sin ^{2}\left(q_{z}+i i\right) d / 2}
$$

so that the stacking-fault energy expression becomes

$$
E_{\mathrm{if}}=\lim _{n \rightarrow 0^{+}} \frac{1}{4 N_{z}^{2}} \sum_{q}^{\prime} \delta_{q_{-}, G} F(q)\left(\frac{A(q)}{\sin ^{2}\left(q_{z}+\mathrm{i} \varepsilon\right) d / 2}+\frac{A^{*}(q)}{\sin ^{2}\left(q_{z}-\mathrm{i} \theta\right) d / 2}\right)
$$

where

$$
A(q)=-\left(f_{1}(q) f_{2}^{*}(q)-\frac{1}{2}\left|f_{1}(q)\right|^{2}-\frac{1}{2} \mid f_{2}(q) !^{2}\right)
$$

In appendix 1 we show that this can be written as

$$
\gamma_{\mathrm{N}}=\frac{N_{\mathrm{c}}}{4 \pi^{2}} \sum_{G} \sum_{l=-x}^{+x}\left(\int_{-x}^{+x} \frac{F(l) \cdot \mathscr{F}^{\mathrm{E}}(l)-F(z) \cdot \mathscr{F}^{\mathrm{E}}(z)}{(z-l)^{2}} \mathrm{~d} z+\left.\pi \frac{\hat{C}}{\hat{\partial} z}\left(F(z) \cdot \mathscr{\mathcal { F }}^{0}(z)\right)\right|_{z=l}\right)
$$

where $\gamma_{* f}$ is the stacking-fault energy per fault ion, $F(z)=F(q(z)), q(z)=|q(z)|$, $q(z)=G_{-}+(2 \pi z / d) \hat{z}$ and

$$
\begin{aligned}
& \mathscr{F}^{\mathrm{F}}(z)=-\left[A(q(z))+A^{*}(q(z))\right] \\
& \mathscr{F}^{0}(z)=-\mathrm{i}\left[A(q(z))-A^{*}(q(z))\right] .
\end{aligned}
$$

As can be seen from equation (15) only the portions of $\tilde{F}^{1}$ that are even in $z$ contribute to the integral sum. and only the portions of $\tilde{H}^{0}$ that are odd in z contribute to the sum. Hence, these will be the only portions of the functions we will need to evaluate equation (15).

Equation (15) is the primary result of this paper. It was obtained by taking the limit $N_{z} \rightarrow \infty$, i.e. the crystal is infinitely long in the $z$ direction with only one fault plane. The other limiting process $s \rightarrow 0^{+}$has also been taken care of exactly. Hence, equation (15) represents an exact result for the stacking-fault energy for a given wavenumber characteristic. The details of the particular stacking-fault structure are embodied in the two functions $\widetilde{F}^{\mathrm{E}}$ and $\mathscr{F}^{0}$. The results obtained previously for the stacking-fault energy of the FCC and HCP faults, using a different mathematical technique for obtaining equations $(11 a)$ and $(11 b)$, can also be cast into the form of equation (15) with a minor change in notation. In fact, equation (15) represents the general form for any stacking-fault energy problem in which the two half-crystals each have perfect crystal structure. So our method is then restricted to the unrelaxed stacking-fault structures.

In general, the wavenumber characteristic is separated into two components: the bandstructure contribution summarising the electron-ion effects and the electrostatic contribution summarising the bare ion-ion effects. We write

$$
F(q)=F^{\mathrm{BS}}(q)+F^{\mathrm{ES}}(q)
$$

The $F^{\mathrm{FS}}(q)$ is known exactly because it is the Coulomb repulsion between the positive ion cores of effective valence $Z^{*}$ :

$$
F^{\mathrm{r}: \mathrm{S}}(q)=2 \pi\left(Z^{*} e\right)^{2} \Omega_{0} q^{2}
$$


where $\Omega_{0}$ is the atomic volume. The contribution to the stacking-fault energy from $F^{\mathrm{ES}}$ can be evaluated separately. In appendix 2 we show that

$$
\gamma_{\perp}^{\mathrm{ES}}=\frac{\pi d N_{\mathrm{c}}}{4 \Omega_{0}}\left(Z^{*} e\right)^{2} \sum_{\bar{G}} \frac{\mathscr{H}\left(G_{\perp} d / N_{亡}\right)}{G_{\perp} \sinh ^{2}\left(G_{\perp} d / 2\right)}
$$

where the $\mathscr{H}$ function is obtained from the $\mathscr{F}^{\mathrm{E}}$ and $\mathscr{F}^{0}$ functions as follows:

$$
\mathscr{H}(\xi)=\mathscr{\mathscr { H }}^{\mathrm{E}}(-\mathrm{i} \xi)+\mathrm{i} \mathscr{\mathscr { H }}^{\mathrm{O}}(-\mathrm{i} \xi)
$$

\section{BCC $\{112$ faults}

In order to calculate the stacking-fault energy for a given fault configuration all we need to do is to find the functions $\mathscr{F}^{\mathrm{E}}$ and $\mathscr{F}^{0}$ which contain the information about the ion positions on each side of the fault, and then evaluate equation (15).

For the $\{112\}$ faults in BCC materials we chose the following basis vectors for the lattice of unit cells:

$$
\boldsymbol{a}=a_{0}(-\hat{i}+\hat{j}) \quad \boldsymbol{b}=a_{0}\left(-\frac{1}{2} \hat{i}-\frac{1}{2} \hat{j}+\frac{1}{2} \hat{k}\right) \quad \boldsymbol{c}=a_{0}(\hat{i}+\hat{j}+2 \hat{k})
$$

where $a_{0}$ is the usual BCC lattice constant. This cell contains six ions. The vectors $\hat{i}, \hat{j}$ and $\hat{k}$ are not the vectors $\hat{x}, \hat{y}$ and $\hat{z}$ of figure 1 . The vector $c$ does lie along $\hat{z}$, however. The reciprocal lattice vectors are

$$
\boldsymbol{A}=\frac{2 \pi}{3 a_{0}}\left(-\frac{3}{2} \hat{i}+\frac{3}{2} \hat{j}\right) \quad \boldsymbol{B}=\frac{2 \pi}{3 a_{0}}(-2 \hat{i}-2 \hat{j}+2 \hat{k}) \quad \boldsymbol{C}=\frac{2 \pi}{3 a_{0}}\left(\frac{1}{2} \hat{i}+\frac{1}{2} \hat{j}+\hat{k}\right)
$$

and therefore, the basal plane reciprocal lattice vectors are

$$
\boldsymbol{G}_{\perp}=h \boldsymbol{A}+k \boldsymbol{B}
$$

for all integers $h$ and $k$.

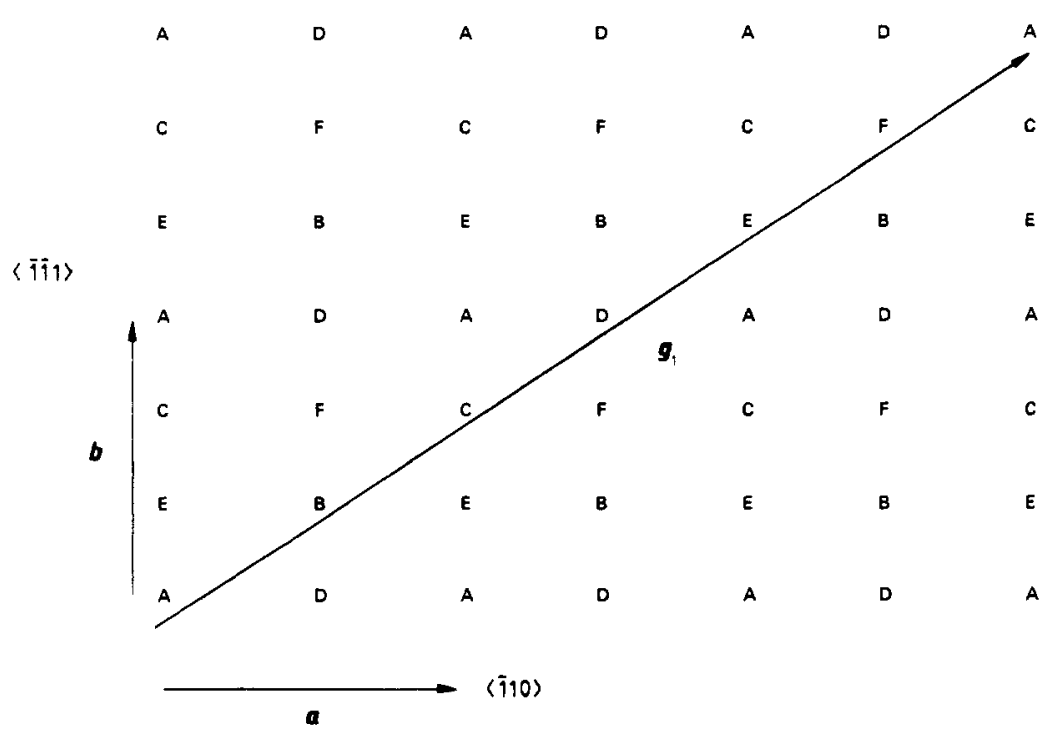

Figure 2. The relative ion positions in the $\{112\}$ plane. The layers are stacked in the sequence A, B. C. . . each $\frac{1}{6}$ the interplanar spacing $d$ above the other. 
Figure 2 shows the relative ionic positions in the $\{112\}$ plane. The planes are stacked one upon the other in the sequences A plane, B plane, C plane, etc. It is convenient to choose the following ion positions for side 1 of the crystal:
A layer: $r_{1}=0$
B layer: $\quad r_{2}=\frac{1}{6}\left(g_{1}+c\right)$
C layer: $r_{3}=\frac{2}{6}\left(g_{1}+c\right)$
D layer: $r_{4}=\frac{3}{5}\left(g_{1}+c\right)$
E layer: $r_{5}=\frac{4}{6}\left(g_{1}+c\right)$
F layer: $\quad r_{6}=\frac{5}{6}\left(g_{1}+c\right)$

where $g_{1}=3 a+2 b$.

Stacking faults are created by disturbing the normal stacking sequence, ABCDEF. Table 1 presents some possible examples of faulted crystals on \{112\} planes. These are the faults that are commonly hypothesised for the simple BCC metals (Hirth and Lothe 1968). Note that the $I_{1}$ fault is geometrically equivalent to two twin faults on adjacent planes. The extrinsic fault is an example of a stacking fault which cannot be handled by the methods of this paper since there is not a single fault plane which divides the crystal into two perfect sequences.

\subsection{Twin faults}

If side 2 is to form a twin fault with respect to side 1, then one possible set of unit-cell ion positions for side 2 is

$$
\begin{aligned}
& r_{1}=\frac{4}{6} g_{1} \\
& r_{2}=\frac{1}{6}\left(-g_{1}+c\right)+\frac{4}{6} g_{1} \\
& r_{3}=\frac{2}{6}\left(-g_{1}+c\right)+\frac{4}{6} g_{1} \\
& r_{4}=\frac{3}{6}\left(-g_{1}+c\right)+\frac{4}{6} g_{1} \\
& r_{5}=\frac{4}{6}\left(-g_{1}+c\right)+\frac{4}{6} g_{1} \\
& r_{6}=\frac{5}{6}\left(-g_{1}+c\right)+\frac{4}{6} g_{1} .
\end{aligned}
$$

Table 1. Possible stacking sequences of 1112 planes in $\mathrm{BCC}$ materials. The asterisk indicates the fault plane.

\begin{tabular}{ll}
\hline Perfect crystal & A BCDEFABCDEF \\
Twin fault & A BCDEFEDCBAF \\
Intrinsic $\left(I_{1}\right)$ fault & A BCDEF*EFABCD \\
Intrinsic $\left(I_{2}\right)$ fault & A BCDEF*CDEFAB \\
Extrinsic fault & A BCDEF*C D*ABCD
\end{tabular}


From equations (5), (19) and (20) we obtain the $f$ functions

$f_{1}=\frac{1}{3}\left[\cos \frac{5}{2}(\zeta+\xi)+\cos \frac{3}{2}(\zeta+\xi)+\cos \frac{1}{2}(\zeta+\xi)\right] \exp \left[-\frac{5}{2} \mathrm{i}(\zeta+\xi)\right]$

$f_{2}=\frac{1}{3}\left[\cos \frac{5}{2}(-\zeta+\xi)+\cos \frac{3}{2}(-\zeta+\xi)+\cos \frac{1}{2}(-\zeta+\xi)\right] \exp \left[-\frac{5}{2} \mathrm{i}(-\zeta+\xi)-4 \mathrm{i} \zeta\right]$

where

$$
\begin{aligned}
& \zeta=\frac{1}{5} G_{-} \cdot g_{1} \\
& \zeta=\frac{1}{6} q_{z} \hat{z} \cdot \hat{c}=2 \pi x / 6 .
\end{aligned}
$$

Then the $\mathscr{F}^{\mathrm{E}}$ and $\mathscr{F}^{0}$ functions are obtained from equations (14) and (16)

$$
\begin{aligned}
& \tilde{\mathscr{H}}^{\mathrm{E}}=\frac{2}{9}(\cos \zeta-1) T_{1}^{2}-\frac{2}{9}(\cos \zeta+1) T_{2}^{2} \\
& \mathscr{F}^{0}=\frac{2}{9}(\sin \zeta)\left(T_{1}^{2}-T_{2}^{2}\right)
\end{aligned}
$$

where

$$
\begin{aligned}
& T_{1}=\cos \frac{5}{2} \zeta \cos \frac{5}{2} \zeta+\cos \frac{3}{2} \zeta \cos \frac{3}{2} \zeta+\cos \frac{1}{2} \zeta \cos \frac{1}{2} \zeta \\
& T_{2}=\sin \frac{5}{2} \zeta \sin \frac{5}{2} \zeta+\sin \frac{3}{2} \zeta \sin \frac{3}{2} \zeta+\sin \frac{1}{2} \zeta \sin \frac{1}{2} \zeta .
\end{aligned}
$$

We can simplify equation (22b) even further. Equation (15) says that only the parts of $\mathscr{F}^{0}$ that are odd in $x$, or equivalently odd in $\xi$, contribute since $F(x)$ is even in $x$. Therefore, the $\mathscr{F}^{0}$ of equation (22b) will contribute nothing since it is even in $\zeta$. Hence, we need only use

$$
\overline{\mathscr{F}}^{0}(\xi)=0
$$

for the twin fault.

The $\mathscr{H}$ function needed for the electrostatic contribution is obtained by the method of equation (18):

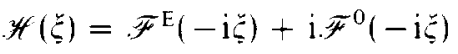

$$
\begin{aligned}
& =\frac{2}{9}(\cos \zeta-1) Q_{1}^{2}+\frac{2}{9}(\cos \zeta+1) Q_{2}^{2}
\end{aligned}
$$

where

$$
\begin{aligned}
& Q_{1}=\cos \frac{5}{2} \zeta \cosh \frac{5}{2} \zeta+\cos \frac{3}{2} \zeta \cosh \frac{3}{2} \zeta+\cos \frac{1}{2} \zeta \cosh \frac{1}{2} \zeta \\
& Q_{2}=\sin \frac{5}{2} \zeta \sinh \frac{5}{2} \zeta+\sin \frac{3}{2} \zeta \sinh \frac{3}{2} \zeta+\sin \frac{1}{2} \zeta \sinh \frac{1}{2} \zeta
\end{aligned}
$$

\subsection{Intrinsic faults}

The intrinsic faults correspond to shifting side 2 with respect to side 1 in the direction of a basal plane vector. A general basal plane direction can be described by $\frac{1}{5} x g_{1}+\frac{1}{2} \beta g_{2}$ where $x$ and $\beta$ are arbitrary and $g_{2}=-a+4 b$ is orthogonal to $g_{1}$. Then $f_{2}$ can easily be described in terms of $f_{1}$

$$
f_{2}=f_{1} \exp \left[-\mathrm{i}\left(x_{\zeta}^{c}+\beta \rho\right)\right]
$$

where

$$
\rho=\frac{1}{2} g_{2} \cdot G_{1}
$$


From this we can obtain the required functions

$$
\begin{aligned}
& \mathscr{F}^{\mathrm{E}}=\frac{2}{9}[\cos (\alpha \zeta+\beta \rho)-1]\left(T_{1}^{2}+T_{2}^{2}\right) \\
& \mathscr{F}^{0}=\frac{4}{9}[\sin (\alpha \zeta+\beta \rho)] T_{1} T_{2} .
\end{aligned}
$$

where the $T_{1}$ and $T_{2}$ terms are the same as those of equations $(23 a)$ and $(23 b)$. In obtaining equation (28) we again made use of the symmetry properties implied by equation (15); namely, that only the even parts of F $^{\mathrm{E}}$ contribute to the integral sum, and only the odd parts of $\mathscr{F}^{0}$ contribute.

The $\mathscr{H}$ function for the electrostatic contribution is

$$
\mathscr{H}=\frac{2}{9}\left[\cos \left(\alpha_{\zeta}^{\circ}+\beta \rho\right)-1\right]\left(Q_{1}^{2}-Q_{2}^{2}\right)+\frac{4}{9}\left[\sin \left(\alpha_{\zeta}+\beta \rho\right)\right] Q_{1} Q_{2}
$$

where $Q_{1}$ and $Q_{2}$ are defined by equation (26). C W Krause (1977, private communication) has corroborated the functional form of equation (29) and also equation (25) using a variation on the method of Fumi and Tosi (1960) for evaluating these electrostatic summations.

By considering various possible values of $x$ and $\beta$ in equations (28) and (29) we can cover all possible shifts of one half-crystal with respect to the other. The $I_{1}$ fault listed in table 1 is described by $x=4$ and $\beta=0$. while the $I_{2}$ fault is given by $x=2 . \beta=0$.

\section{Numerical results}

The integration of equation (15) was performed in the same manner as our previous work (Devlin 1974). For each of the metals we integrated out to a value of $q$ where $F(q)$ was negligible. The tabular data for $F(q)$ was interpolated with a six-point Lagrange formula.

For the metals $\mathrm{Li}, \mathrm{Na}, \mathrm{K}, \mathrm{Rb}, \mathrm{Cs}, \mathrm{Ca}, \mathrm{Sr}$ and $\mathrm{Ba}$ we used the model potentials developed by AW. The potentials obtained by the AW method for the metals Al, Mg and Be were highly successful in our study of stacking faults in these FCC and HCP metals. Because the model potential theory is just as applicable to the metals $\mathrm{Li}, \mathrm{Na} . \mathrm{K}$ and $\mathrm{Ca}$, we would expect the potentials so generated for these metals to also be valid to the same extent. For the higher atomic number metals, $\mathrm{Rb}, \mathrm{Sr}, \mathrm{Cs}$ and $\mathrm{Ba}$, the model potentials are less reliable because of the localised $d$ states which are neglected in model potential theory.

We have also used the Moriarty (1972) potentials for $\mathrm{Ca}, \mathrm{Sr}$ and $\mathrm{Ba}$. These potentials were obtained from a generalisation of the pseudopotential theory which incorporates the localised $\mathrm{d}$ states to some extent.

Table 2 summarises the results for the stacking-fault energies of the alkaline earth metals $\mathrm{Ca}, \mathrm{Sr}$ and $\mathrm{Ba}$. The Moriarty and $\mathrm{AW}$ potentials agree reasonably well for the metals $\mathrm{Ca}$ and $\mathrm{Sr}$. There is a substantial discrepancy in the results of the two potentials in the case of the heaviest alkaline earth $\mathrm{Ba}$. The $\mathrm{AW} \mathrm{Ba}$ twin-fault energy is nearly twice that for the Moriarty twin fault because of the difference in signs for the $i_{B s}$ term. In all three metals $i_{1}=i_{1}=2 ;$. Because the $I_{1}$ fault is geometrically equivalent to two twin faults on adjacent planes and energetically $i_{t}=2 i_{i}$, we are led to the conclusion that twin faults in these metals do not interact strongly with each other. This is consistent with the work done on the FCC stacking-fault interactions (Devlin 1975). 
Table 2. Stacking-fault energies of $\mathrm{Ca}$, Sr and $\mathrm{Ba}$. The Moriarty (1972) and AW potentials were used. Here $\gamma_{\mathrm{FS}}$ is the electrostatic contribution, $\gamma_{\mathrm{BS}}$ is the bandstructure contribution, and $\gamma_{\text {totit }}$ is the sum of these. These stacking-fault energies are all reported in units of erg $\mathrm{cm}^{-2}$.

\begin{tabular}{lrrr}
\hline & \multicolumn{1}{c}{$\gamma_{\text {t.S }}$} & $\gamma_{\text {HS }}$ & $\eta_{\text {totul }}$ \\
\hline Twin faults & & & \\
Ca (Moriarty) & 114.3 & -30.1 & 84.2 \\
Sr (Moriarty) & 96.6 & -27.2 & 69.4 \\
Ba (Moriarty) & 101.5 & -45.1 & 56.4 \\
Ca (AW) & 100.1 & -28.6 & 71.5 \\
Sr (AW) & 79.4 & -12.2 & 67.2 \\
Ba (AW) & 79.6 & +17.5 & 97.1 \\
I faults & & & \\
Ca (Moriarty) & 230,5 & -61.3 & 169.2 \\
Sr (Moriarty) & 194.8 & -54.7 & 140.1 \\
Ba (Moriarty) & 204.8 & -79.7 & 125.1 \\
Ca (AW) & 201.8 & -63.3 & 138.5 \\
Sr (Aw) & 160.1 & -32.6 & 127.5 \\
Ba (Aw) & 160.5 & +26.9 & 187.4 \\
I faults & & & \\
Ca (Moriarty) & 228.7 & -61.3 & 167.4 \\
Sr (Moriarty) & 193.3 & -54.7 & 138.6 \\
Ba (Moriarty) & 203.1 & -79.7 & 123.4 \\
Ca (AW) & 200.2 & -63.4 & 136.8 \\
Sr (AW) & 158.8 & -32.6 & 126.2 \\
Ba (AW) & 159.2 & +27.0 & 186.2 \\
\hline
\end{tabular}

Table 3. Stacking-fault energies of the alkali metals. The Aw potential was used in each case. Here $\gamma_{\text {ES }}$ is the electrostatic contribution, $\gamma_{\text {BS }}$ is the bandstructure contribution, and $\gamma_{\text {tolal }}$ is the sum of these. All results are reported in units of erg $\mathrm{cm}^{-2}$.

\begin{tabular}{llrr}
\hline & $\gamma_{\text {ES }}$ & $\gamma_{\text {BS }}$ & $\gamma_{\text {hotal }}$ \\
\hline Twin faults & & & \\
$\quad \mathrm{Li}$ & 46.9 & 31.6 & 78.5 \\
$\mathrm{Na}$ & 27.4 & -1.7 & 25.7 \\
$\mathrm{~K}$ & 15.7 & -0.3 & 15.4 \\
$\mathrm{Rb}$ & 13.0 & -0.9 & 12.1 \\
$\mathrm{Cs}$ & 11.0 & -0.6 & 10.4 \\
$\mathrm{I}_{1}$ faults & & & \\
$\mathrm{Li}$ & 94.7 & 47.3 & 142.0 \\
$\mathrm{Na}$ & 55.2 & -4.1 & 51.1 \\
$\mathrm{~K}$ & 31.6 & -1.3 & 30.3 \\
$\mathrm{Rb}$ & 26.3 & -2.4 & 23.9 \\
$\mathrm{Cs}$ & 22.3 & -1.8 & 20.5 \\
$\mathrm{I}_{2}$ faults & & & \\
$\mathrm{Li}$ & 93.9 & 47.3 & 141.2 \\
$\mathrm{Na}$ & 54.8 & -4.2 & 50.6 \\
$\mathrm{~K}$ & 31.3 & -1.3 & 30.0 \\
$\mathrm{Rb}$ & 26.1 & -2.4 & 23.7 \\
$\mathrm{Cs}$ & 22.1 & -1.8 & 20.3 \\
\hline
\end{tabular}


Table 3 summarises the results for the stacking-fault energies of the alkali metals. Again, we see that $\gamma_{1_{1}} \simeq \gamma_{\mathrm{I}_{2}} \simeq 2 \gamma_{\mathrm{T}}$ indicating weakly interacting twin faults. Note that for the alkalis the electrostatic term is the main contributor to the stacking-fault energy except in the case of $\mathrm{Li}$.

The results reported for the intrinsic faults $I_{1}$ and $I_{2}$ in tables 2 and 3 are the results of the ideal intrinsic faults as listed in table 1 . We have also considered arbitrary shifts of one half-crystal with respect to another. A general shift (see $\$ 3.2)$ is described by $\delta=\frac{1}{6} \alpha g_{1}+\frac{1}{2} \beta g_{2}$ where the $\alpha$ and $\beta$ are arbitrary. Each pair of $\alpha, \beta$ produces a stacking-fault energy $\gamma(\alpha, \beta)$. A plot of $\gamma(\alpha, \beta)$ in three space yields a two-dimensional surface called the gamma surface. An investigation of the gamma surface yields the possible (meta)stable stacking faults. In figures 3 and 4 we have plotted $\gamma(\alpha, \beta)$ for certain directions in the $(\alpha, \beta)$ space. These plots are for Na with the AW potential. The plots suggest minima in the $\gamma$ values. The minima in figure 3 are in fact true minimas, not saddle points. These minimas are located close to the ideal stacking faults of table 1 . The $I_{1}$ fault is ideal when $\alpha=4, \beta=0$. We find the minima nearest this to be at $\alpha \simeq 4.15, \beta \simeq 0.0$. The value of $\gamma$ at the minima is $14.9 \mathrm{erg} \mathrm{cm}^{-2}$ while the ideal structure for $I_{1}$ yields a value of $51.1 \mathrm{erg} \mathrm{cm}^{-2}$. Hence, $\gamma$ is sensitive to small displacements about the minima. The ideal $\mathrm{I}_{2}$ fault corresponds to $\alpha=2, \beta=0$. The minima nearest this occurs at $\alpha \simeq 2.00, \beta \simeq 0.15$. The value of $\gamma$ at the minima is -42.6 while the ideal structure has $y_{1}=50.6$. A negative fault energy at the $I_{2}$ minima indicates that the BCC phase is unstable with respect to this type of deformation. Thus, the AW potential does not predict the ideal BCC structure as the most stable phase for $\mathrm{Na}$. A separate structural energy calculation for $\mathrm{Na}$ for the perfect crystal structures shows that the HCP structure (with an ideal axial ratio) is preferred over the FCC and BCC structures. In such a case then it is not too surprising that the BCC structure with one defect has less energy than the perfect BCC structure.

A negative $\gamma$ value also occurs near the point $\alpha=5.85, \beta=0$ (see figure 3 ). In fact, the slope of the $\gamma(\alpha, 0)$ versus $\alpha$ curve is positive in the region $\alpha=6$ (or equivalently

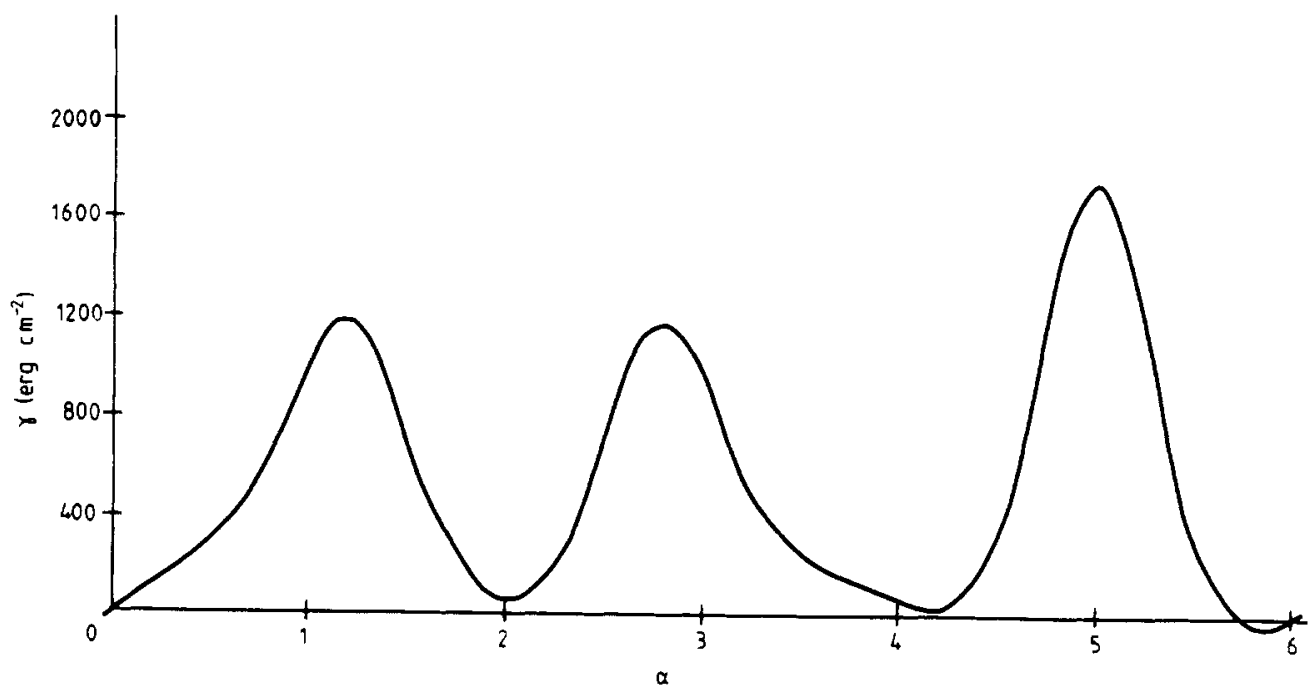

Figure 3. Plot of the $\gamma(\alpha, \beta)$ surface along the $\beta=0$ direction. For sodium with the Aw potential. 


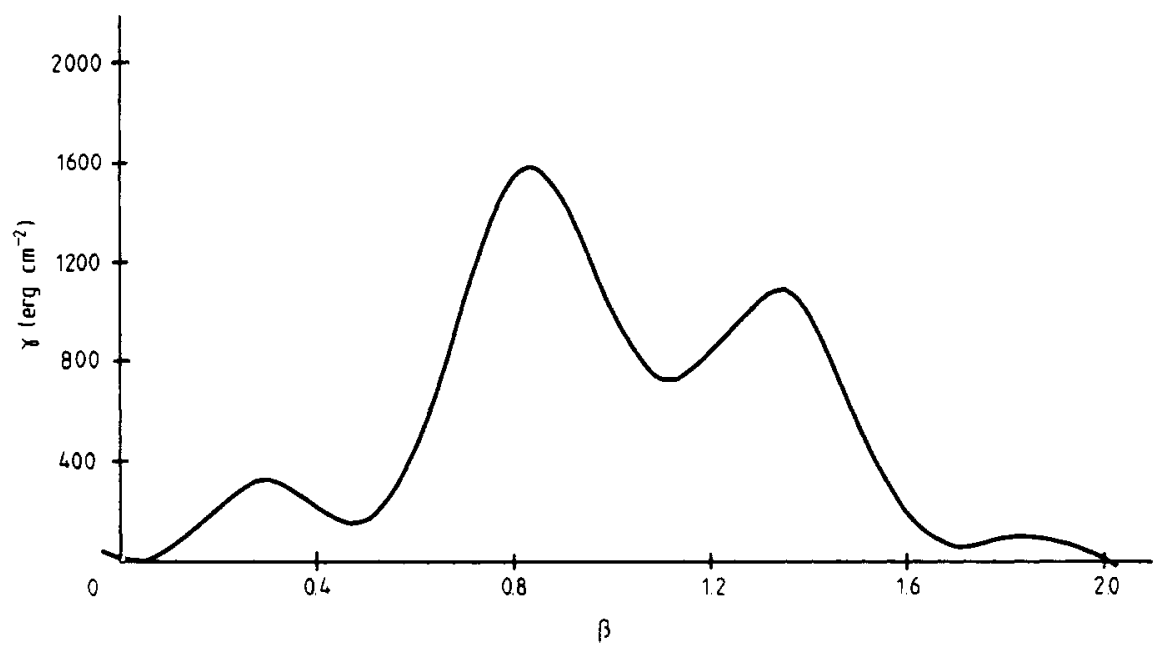

Figure 4. Plot of the $;(x, \beta)$ surface along the $x=0$ direction. For sodium with the AW potential.

$\alpha=0$ ). So again, the BCC phase is unstable with respect to certain distortions when the Aw potential is used. This negative value of $\gamma$ for small positive displacements along $\boldsymbol{g}_{1}$ was noted for all of the eight metals reported here even when two different potentials were available. Coinciding with this is the fact that our separate structural calculations show that neither the AW nor Moriarty potentials predict BCC as the preferred phase with the sole exception of $\mathrm{Li}$. In the case of $\mathrm{Li}$, the BCC phase is slightly preferred over the FCC and HCP phase, but this particular preference does not in any way prove that the BCC phase has the absolute lowest energy of all possible crystal structures. Such a search for the absolute lowest energy structure would be computationally unfeasible. Our conclusion then is that negative fault energies are indicative of potentials that cannot correctly predict the known crystal structures. Such predictions are extremely difficult to make since the energy difference between the phases is one part in $10^{5}$ of the total structure-dependent energy and would indeed be a severe test of any model or pseudopotential.

Notwithstanding negative fault energies, the ability of these potentials to predict metastable faults seems assured. The minima in $\gamma(\alpha, \beta)$ that we have found for these BCC faults are well-defined and in the expected regions. The actual values of $\gamma$, however, are suspect. Ba is a good example of this effect. Both the AW and Moriarty potential predict that the $I_{1}$ fault minima would occur approximately at the $\alpha=4.15$, $\beta=0$ point; however, the Moriarty potential yields $\hat{i}=-5.5 \mathrm{erg} \mathrm{cm}^{-2}$ at that point, while the Aw potential yields $y=100.1 \mathrm{erg} \mathrm{cm}^{-2}$.

\section{Summary and conclusions}

We have developed a general procedure for calculating the stacking-fault energy of unrelaxed stacking faults, i.e. faults which are perfect crystal structures up to the fault plane. The only approximations that enter into this procedure enter through the wavenumber characteristic. 
Our procedure was used to study the $\{112\}$ stacking faults in BCC structures. The AW potentials were used to study the BCC phases of $\mathrm{Li}, \mathrm{Na}, \mathrm{K}, \mathrm{Rb}, \mathrm{Cs}, \mathrm{Ca}, \mathrm{Ba}$ and $\mathrm{Sr}$, while the Moriarty potentials were used for $\mathrm{Ca}, \mathrm{Ba}$ and $\mathrm{Sr}$. In each of the metals (and for each of the potentials) we noted stable stacking-fault configurations in the vicinity of the predicted structures. The actual values of the stacking-fault energies cannot be compared directly with experiment for two reasons. First, two of these metals (Ca and Sr) do not even exist in the BCC phase. Second, with the exception of Li, neither potential predicts $\mathrm{BCC}$ to be the most stable crystal phase, and therefore, some of the stacking-fault energies turn out to be negative. Hence, the model and pseudopotentials are inadequate for these metals when doing structural studies on the BCC phase.

The possible role played by relaxing the ions in the region of the fault to produce realistic configurations is still open to question. Our fault energies for the unrelaxed faults would clearly be upper bounds on the relaxed fault energy since any relaxation would be to minimise the energy.

Since we already have negative energies for some of the $\mathrm{BCC}$ faults, any relaxation effects that we might introduce would not be very effective in adding to our knowledge of the true structure and energy of the BCC faults.

\section{Acknowledgment}

The author wishes to thank Dr C W Krause for valuable conversations during the course of this work.

\section{Appendix 1}

Here we derive equation (15) from equation (13).

Writing $q_{z}=2 \pi z / d, \sigma=\varepsilon d / 2 \pi$, and converting the sum over $q_{z}$ to an integral over zwe have

$E_{\mathrm{vf}}=\lim _{\sigma \rightarrow 0^{-}} \frac{1}{4 N_{z}} \frac{5}{G} \int_{-x}^{-x} \mathrm{~d} z F\left(\boldsymbol{G}_{\perp}, q_{z}(z)\right)\left(\frac{A\left(\boldsymbol{G}_{-}, q_{z}(z)\right)}{\sin ^{2}(\pi z+\mathrm{i} \pi \sigma)}+\frac{A^{*}\left(\boldsymbol{G}_{\perp}, q_{z}(z)\right)}{\sin ^{2}(\pi z-\mathrm{i} \pi \sigma)}\right)$

Hereafter. we will only show explicitly the $z$ dependence of $F\left(G_{\perp}, q_{z}(z)\right)$ and $A\left(G_{+}, q_{z}(z)\right)$. Now using the identity

$$
\frac{1}{\sin ^{2} \pi(z+\mathrm{i} \sigma)}=\frac{1}{\pi^{2}} \sum_{t=-x}^{+\infty} \frac{1}{(z-1+\mathrm{i} \sigma)^{2}}
$$

and setting $\Phi(z)=F(z) A(z)$ we have

$$
E_{\mathrm{S}}=\lim _{\sigma \rightarrow 0} \frac{1}{4 \pi^{2} N_{z}} \sum_{G} \sum_{l=-x}^{+x} \int_{-x}^{+x} \mathrm{~d} z\left(\frac{\Phi(z)}{(z-l+\mathrm{i} \sigma)}+\mathrm{CC}\right)
$$

where $C C$ is the complex conjugate. Now we add and subtract the same terms to the equation

$$
\begin{aligned}
E_{\mathrm{df}}=\lim _{\sigma \rightarrow 0^{-}} \frac{1}{4 \pi^{2} N_{z}} \sum_{G} \sum_{l=-x}^{+x} \int_{-x}^{+x} \mathrm{~d} z\left(\frac{\Phi(z)-\Phi(l)}{(z-l+\mathrm{i} \sigma)^{2}}+\mathrm{CC}\right) \\
+\lim _{\sigma \rightarrow 0^{-}} \frac{1}{4 \pi^{2} N_{z}} \sum_{G} \sum_{l=-x}^{+x} \int_{-x}^{+x} \mathrm{~d} z\left(\frac{\Phi(l)}{(z-l+\mathrm{i} \sigma)^{2}}+C C\right)
\end{aligned}
$$


For the $(z-l+\mathrm{i} \sigma)^{-2}$ term we use the following identity in the first part of $E_{\mathrm{s}}$

$$
\lim _{\sigma \rightarrow 0^{+}} \frac{1}{(z-l+\mathrm{i} \sigma)^{2}}=P \frac{1}{(z-l)^{2}}+\mathrm{i} \pi \delta^{\prime}(z-l)
$$

where $P$ represents the principal part integral and $\delta^{\prime}$ represents the derivative of the Dirac delta function. Then equation (A.3) becomes

$$
\begin{aligned}
E_{\mathrm{v}}=\frac{1}{4 \pi^{2} N_{z}} & \sum_{G_{-}} \sum_{l=-\infty}^{+\infty}\left(P \int_{-\infty}^{+\infty} \frac{(\Phi(z)-\Phi(l))+\mathrm{CC}}{(z-l)^{2}} \mathrm{~d} z\right. \\
& \left.+\int_{-\infty}^{+\infty}\left[(\Phi(z)-\Phi(l)) \mathrm{i} \pi \delta^{\prime}(z-l)+\mathrm{CC}\right] \mathrm{d} z\right)+E_{\cdot \mathrm{i}}^{(2)}
\end{aligned}
$$

where

$$
E_{* f}^{(2)}=\lim _{\sigma \rightarrow 0^{+}} \frac{1}{4 \pi^{2} N_{z}} \sum_{\sigma_{s}} \sum_{l=-\infty}^{+\infty} \int_{-\infty}^{+\infty} \mathrm{d} z\left(\frac{\Phi(l)}{(z-l+\mathrm{i} \sigma)^{2}}+\mathrm{CC}\right)
$$

Now we note that

$$
\begin{aligned}
& \Phi(z)+\Phi^{*}(z)=F(z)\left(A(z)+A^{*}(z)\right)=-F(z) \cdot \bar{F}^{\mathrm{E}}(z) \\
& \Phi(z)-\Phi^{*}(z)=F(z)\left(A(z)-A^{*}(z)\right)=\mathrm{i} F(z) \cdot \mathscr{F}^{0}(z)
\end{aligned}
$$

where the $\tilde{\mathscr{H}}^{1}$ and $\tilde{\mathscr{H}}^{0}$ functions are as defined in equations $(16 a)$ and $(16 b)$, respectively. Then equation (A.5) becomes

$$
\begin{aligned}
& E_{-}=\frac{1}{4 \pi^{2} N_{z}} \sum_{G_{-} l} \sum_{-}^{+\infty}\left(P \int_{-x}^{+x} \frac{F(l) \mathscr{F} \mathrm{E}^{2}(l)-F(z) \mathscr{F}^{\mathrm{E}}(z)}{(z-l)^{2}} \mathrm{~d} z\right. \\
& \left.-\int_{-}^{+\infty} \pi\left(F(z) \cdot \overline{\mathscr{H}}^{0}(z)-F(l) \tilde{\mathscr{F}}^{0}(l)\right) \delta^{\prime}(z-l) \mathrm{d} z\right)+E_{\triangleleft !}^{(2)} .
\end{aligned}
$$

By the usual properties of the Dirac delta function, this can be written as

$$
\begin{gathered}
E_{\mathrm{S}}=\frac{1}{4 \pi^{2} N_{z}} \sum_{G} \sum_{l}^{+\infty},\left(P \int_{-x}^{+\infty} \frac{F(l) \mathscr{F} \mathrm{E}(l)-F(z) \mathscr{F}^{\mathrm{E}}(z)}{(z-l)^{2}} \mathrm{~d} z\right. \\
\left.+\left.\pi \frac{\hat{c}}{\hat{c} z} F(z) \cdot \mathscr{\mathscr { F }}^{0}(z)\right|_{z=l}\right)+E_{\mathrm{sf}}^{(2)} .
\end{gathered}
$$

The principal value notation on the integral above is unnecessary at this point since the integral is analytic at $z=l$. To get $\gamma_{1}$, the stacking-fault energy per fault ion, we multiply by $N_{\mathrm{c}} N_{2}$

$$
\begin{gathered}
\gamma_{s \mathrm{f}}=\frac{N_{\mathrm{c}}}{4 \pi^{2}} \sum_{\sigma} \sum_{l=-x}^{+x}\left(\int_{-x}^{+x} \frac{F(l) \cdot \mathscr{F}^{\mathrm{E}}(l)-F(z) \cdot \mathscr{F}^{\mathrm{E}}(z)}{(z-l)^{2}} \mathrm{~d} z\right. \\
\left.+\left.\pi \frac{\hat{c}}{\partial z} F(z) \cdot \mathscr{F}^{0}(z)\right|_{z=l}\right)+N_{\mathrm{v}} N_{z} E_{\cdot .}^{(2)} .
\end{gathered}
$$

Equation (A.6) is the same as equation (15) except for the term $E_{s}^{(2)}$. We shall show 
that $E_{\mathrm{sf}}^{(2)}$ is zero. The integration over $z$ in the definition of $E_{\mathrm{sf}}^{(2)}$ can be performed as follows

$$
E_{\mathrm{sf}}^{(2)}=\lim _{\sigma \rightarrow 0^{+}} \frac{1}{4 \pi^{2} N_{z}} \sum_{G_{\perp}} \sum_{l=-\infty}^{+\infty} \sum_{n=-\infty}^{+\infty} \int_{-1 / 2}^{+1 / 2} \mathrm{~d} z\left(\frac{\Phi(l)}{(z+n-l+\mathrm{i} \sigma)^{2}}+\mathrm{CC}\right) .
$$

However

$$
\sum_{n=-\infty}^{+\infty} \frac{1}{(z+n-l+\mathrm{i} \sigma)^{2}}=\frac{\pi^{2}}{\sin ^{2} \pi(z-l+\mathrm{i} \sigma)}=\frac{\pi^{2}}{\sin ^{2} \pi(z+\mathrm{i} \sigma)}
$$

so

$$
E_{\mathrm{sf}}^{(2)}=\lim _{\sigma \rightarrow 0^{+}} \frac{1}{4 N_{z}} \sum_{G_{1}} \sum_{l=-\infty}^{+\infty} \int_{-1 / 2}^{+1 / 2} \mathrm{~d} z\left(\frac{\Phi(l)}{\sin ^{2} \pi(z+\mathrm{i} \sigma)}+\mathrm{cc}\right) .
$$

By choosing a contour in the upper half of the complex plane, it is possible to show that

$$
\lim _{\sigma \rightarrow 0^{+}} \int_{-1 / 2}^{+1 / 2} \mathrm{~d} z \frac{1}{\sin ^{2} \pi(z+\mathrm{i} \sigma)}=0 .
$$

Hence, $E_{\mathrm{si}}^{(2)}=0$ and therefore equation (A.6) leads to equation (15).

\section{Appendix 2}

Here we derive equation (17) from equation (15) for the case of the electrostatic wavenumber characteristic

$$
F^{\mathrm{ES}}(q)=\frac{2 \pi\left(Z^{*} e\right)^{2}}{\Omega_{0} q^{2}}=\frac{2 \pi\left(Z^{*} e\right)^{2}}{\Omega_{0}\left[G_{\perp}^{2}+(2 \pi z / d)^{2}\right]} .
$$

We begin by defining

$$
\mathscr{F}(z)=\mathscr{F}^{\mathrm{E}}(z)+\mathrm{i}^{\circ} \mathrm{O}(z)
$$

and, with the use of equation (A.4), equation (15) can be written as

$$
\gamma_{\mathrm{s} f}^{\mathrm{ES}}=\lim _{\sigma \rightarrow 0^{+}} \frac{N_{\mathrm{c}}}{8 \pi^{2}} \sum_{\sigma_{\perp}} \sum_{l=-\infty}^{+\infty}\left(\int_{-\infty}^{+\infty} \mathrm{d} z \frac{F^{\mathrm{ES}}(l) \mathscr{F}(l)-F^{\mathrm{ES}}(z) \mathscr{F}(z)}{[z-(l+\mathrm{i} \sigma)]^{2}}+\mathrm{cc}\right) .
$$

Now the $\mathscr{F}(z)$ function, since it is obtained from the $f_{1}$ and $f_{2}$ functions of equation (5), can, in general, be expressed as a sum of exponentials

$$
\mathscr{F}(z)=\sum_{j} a_{j} \exp \left(\mathrm{i} b_{j} z\right)
$$

where $a_{j}$ and $b_{j}$ are constants. 
We separate $\tilde{F}(z)$ into two parts, one with positive (or zero) $b_{j}$ and one with negative $b_{j}$ :

$$
\begin{aligned}
& \mathscr{F}^{+}(z) \equiv \sum_{\substack{j \\
b_{j} \geqslant 0}} a_{j} \exp \left(\mathrm{i} b_{j} z\right) \\
& \mathscr{F}^{-}(z) \equiv \sum_{\substack{j \\
b_{j}<0}} a_{j} \exp \left(\mathrm{i} b_{j} z\right) .
\end{aligned}
$$

Now we define

$$
\begin{aligned}
& g^{+}(z)=\frac{1}{[z-(l+\mathrm{i} \sigma)]^{2}}\left(\frac{\mathscr{F}^{+}(z)}{G_{\perp}^{2}+(2 \pi z / d)^{2}}-\frac{\mathscr{F}^{+}(l)}{G_{\perp}^{2}+(2 \pi l / d)^{2}}\right) \\
& g^{-}(z)=\frac{1}{[z-(l+\mathrm{i} \sigma)]^{2}}\left(\frac{\mathscr{F}^{-}(z)}{G_{\perp}^{2}+(2 \pi z / d)^{2}}-\frac{\mathscr{F}^{-}(l)}{G_{\perp}^{2}+(2 \pi l / d)^{2}}\right) .
\end{aligned}
$$

Hence

$\gamma_{\mathrm{sf}}^{\mathrm{ES}}=\lim _{\sigma \rightarrow 0^{+}} \frac{-N_{\mathrm{c}}}{8 \pi^{2}} \frac{2 \pi\left(Z^{*} e\right)^{2}}{\Omega_{0}} \sum_{G_{-}} \sum_{l=-\infty}^{+\infty} \int_{-\infty}^{+\infty}\left[\left(g^{+}(z)+g^{-}(z)\right)+\mathrm{Cc}\right] \mathrm{d} z$.

The integrals in equation (A.9) can be performed by contour integration. The function $g^{+}(z)$ is bounded in the upper half plane, while $g^{-}(z)$ is bounded in the lower half plane. The functions have poles at $z=l+\mathrm{i} \sigma$ (second order) and $z= \pm(2 \pi \mathrm{i} / d) G_{\perp}$ (first order). The integral for $g^{-}(z)$ is easily obtained

$$
\int_{-\infty}^{+\infty} g^{-}(z) \mathrm{d} z=\frac{d}{2 G_{\perp}} \frac{\mathscr{F}^{-}\left(-\mathrm{i} G_{\perp} d / 2 \pi\right)}{\left(l+\mathrm{i} \sigma+\mathrm{i} G_{\perp} d / 2 \pi\right)^{2}}
$$

and for $g^{+}(z)$

$$
\int_{-x}^{+x} g^{+}(z) \mathrm{d} z=\frac{d}{2 G_{\perp}} \frac{\mathscr{F}^{+}\left(\mathrm{i} G_{-} d / 2 \pi\right)}{\left(l+\mathrm{i} \sigma-\mathrm{i} G_{\perp} d / 2 \pi\right)^{2}}+2 \pi \mathrm{i} \frac{\hat{c}}{\partial z}\left(\frac{\mathscr{F}^{+}(z)}{G_{\perp}^{2}+(2 \pi z / d)^{2}}\right)_{z=1+\mathrm{i} \sigma}
$$

Then equation (A.9) becomes

$$
\begin{aligned}
\gamma_{\mathrm{s} f}^{\mathrm{ES}}=\lim _{\sigma \rightarrow 0^{+}} \frac{-N_{\mathrm{c}}}{8 \pi^{2}} \frac{2 \pi\left(Z^{*} e\right)^{2}}{\Omega_{0}} \sum_{G_{-}} \sum_{l=-\infty}^{+\infty}\left\{\left[\frac{d}{2 \pi} \frac{\mathscr{F}^{-}\left(-\mathrm{i} G_{\perp} d / 2 \pi\right)}{\left(l+\mathrm{i} \sigma+\mathrm{i} G_{\perp} d / 2 \pi\right)^{2}}\right.\right. \\
\left.\left.+\frac{d}{2 \pi} \frac{\mathscr{F}^{+}\left(\mathrm{i} G_{\perp} d / 2 \pi\right)}{\left(l+\mathrm{i} \sigma-\mathrm{i} G_{\perp} d / 2 \pi\right)^{2}}+2 \pi \mathrm{i} \frac{\partial}{\partial z}\left(\frac{\mathscr{F}^{+}(z)}{G_{\perp}^{2}+(2 \pi z / d)^{2}}\right)_{z=l+\mathrm{i} \sigma}\right]+\mathrm{CC}\right\}
\end{aligned}
$$

which with the use of equation (A.2) yields

$$
\begin{gathered}
\gamma_{\mathrm{sf}}^{\mathrm{ES}}=\frac{N_{\mathrm{c}}}{8 \pi} \frac{2 \pi\left(Z^{*} e\right)^{2}}{\Omega_{0}} \sum_{G_{-}}\left\{\left[\frac{\pi^{2} d}{2 G_{\perp}}\left(\frac{\mathscr{F}^{+}\left(\mathrm{i} G_{\perp} d / 2 \pi\right)+\mathscr{F}^{-}\left(-\mathrm{i} G_{\perp} d / 2 \pi\right)}{\sinh ^{2}\left(G_{\perp} d / 2\right)}\right)\right.\right. \\
\left.\left.+2 \pi \mathrm{i} \sum_{l=-\infty}^{+\infty} \frac{\partial}{\partial z}\left(\frac{\mathscr{F}^{+}(z)}{G_{\perp}^{2}+(2 \pi z / d)^{2}}\right)_{z=1}\right]+\mathrm{CC}\right\} .
\end{gathered}
$$


Now we define

$$
h(z)=[\exp (2 \pi \mathrm{i} z)-1]^{-1} \frac{\hat{c}}{\partial z}\left(\frac{\tilde{F}^{+}(z)}{G_{\perp}^{2}+(2 \pi z / d)^{2}}\right) .
$$

This function is bounded everywhere at infinity so that

$$
\oint h(z) \mathrm{d} z=0
$$

where the contour encloses the whole complex plane. Then the sum of the residues of this function is zero. This leads to the following identity

$2 \pi \mathrm{i} \sum_{l=-\infty}^{+\infty} \frac{\hat{c}}{\hat{c} z}\left(\frac{\tilde{F}^{+}(z)}{G_{-}^{2}+(2 \pi z / d)^{2}}\right)_{z=l}=\frac{d \pi^{2}}{2 G_{\perp}} \frac{\mathscr{\mathscr { F }}^{+}\left(\mathrm{i} G_{\perp} d / 2 \pi\right)-\mathscr{F}^{+}\left(-\mathrm{i} G_{\perp} d / 2 \pi\right)}{\sinh ^{2}\left(G_{\perp} d / 2\right)}$.

Then equation (A.12) becomes

$$
\begin{array}{r}
\gamma_{\mathrm{sf}}^{\mathrm{ES}}=\frac{N_{\mathrm{c}}}{8 \pi^{2}} \frac{2 \pi\left(Z^{*} e\right)^{2}}{\Omega_{0}} \sum_{G}\left\{\left[\frac{d \pi^{2}}{2 G_{\perp}}\left(\frac{\mathscr{F}^{+}\left(\mathrm{i} G_{\perp} d / 2 \pi\right)+\mathscr{F}^{-}\left(-\mathrm{i} G_{\perp} d / 2 \pi\right)}{\sinh ^{2}\left(G_{\perp} d / 2\right)}\right)\right.\right. \\
\left.\left.-\frac{d \pi^{2}}{2 G_{-}}\left(\frac{\mathscr{F}^{+}\left(\mathrm{i} G_{\perp} d / 2 \pi\right)-\mathscr{F}^{+}\left(-\mathrm{i} G_{\perp} d / 2 \pi\right)}{\sinh ^{2}\left(G_{\perp} d / 2\right)}\right)\right]+\mathrm{CC}\right\} .
\end{array}
$$

Since $\mathscr{F}^{-*}(x)=\mathscr{F}^{-}(-x), \mathscr{F}^{+*}(x)=\mathscr{F}^{+}(-x)$, we have our final result

$$
\gamma_{\mathrm{s} f}^{\mathrm{ES}}=\frac{\pi d N_{\mathrm{c}}\left(Z^{*} e\right)^{2}}{4 \Omega_{0}} \sum_{G} \frac{\mathscr{F}^{+}\left(-\mathrm{i} G_{\perp} d / 2 \pi\right)+\mathscr{F}^{-}\left(-\mathrm{i} G_{\perp} d / 2 \pi\right)}{G_{\perp} \sinh ^{2}\left(G_{\perp} d / 2\right)} .
$$

In terms of the $\xi$ variable, $\xi=2 \pi z / N_{\mathrm{c}}$, we have

$$
\gamma_{\mathrm{sf}}^{\mathrm{ES}}=\frac{\pi d N_{\mathrm{c}}\left(Z^{*} e\right)^{2}}{4 \Omega_{0}} \sum_{G} \frac{\mathscr{F}^{\mathrm{E}}\left(-\mathrm{i} G_{\perp} d / N_{\mathrm{c}}\right)+\mathrm{i} \mathscr{F}^{0}\left(-\mathrm{i} G_{\perp} d / N_{c}\right)}{\sinh ^{2}\left(G_{\perp} d / 2\right)}
$$

where we have reverted back to the $\mathscr{F}^{\mathrm{E}}$ and $\mathscr{F}^{\mathrm{O}}$ functions. Hence, the definition in equation (18) leads to equation (17).

\section{References}

Appapillai M and Williams A R 1973 J. Phy's. F: Met. Phys. 3 759-71

Basinski Z S, Duesbery M S and Taylor R 1970 Phil. Mag. 21 1201-21

Bollmann W 1970 Crystal Defects and Crystalline Interfaces (Berlin: Springer)

Devlin J F 1974 J. Phys. F: Met. Phys. 4 1865-81

- 1975 Phys. Lett. 51A 131-2

Eichler H and Pegel B 1969 Phys. Status Solidi 35 333-8

Fumi F G and Tosi M P 1960 Phys. Rer. 117 1466-8

Hirth J P and Lothe J 1968 Theory of Dislocations (New York: McGraw-Hill)

Hodges C H 1967 Phil. Mag. 134 371-92

Moriarty J A 1972 Phys. Ret. B 6 4445-58

Rao P V S 1975 J. Phys. F: Met. Phys. 5 611-28

Simon J P 1979 J. Phys. F: Met. Phys. 9 425-9

Vitek V 1968 Phil. Mag. 18 773-85 